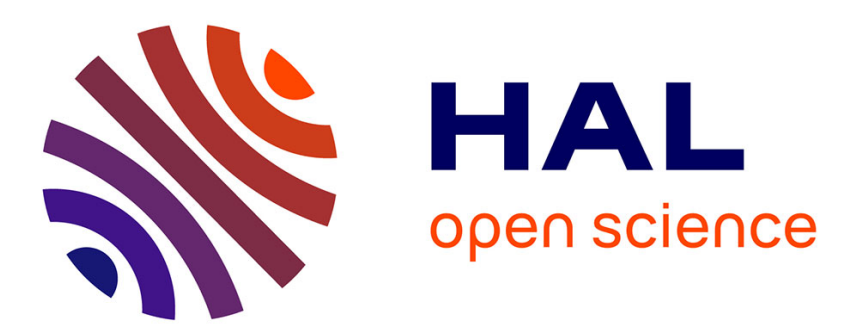

\title{
Les quarantaines au Moyen-Orient : vecteurs ambigus de la modernité médicale (XIXe-XXe siècles) \\ Sylvia Chiffoleau
}

\section{To cite this version:}

Sylvia Chiffoleau. Les quarantaines au Moyen-Orient: vecteurs ambigus de la modernité médicale (XIXe-XXe siècles). Modernity and Modernization of Medicine in the Ottoman Empire and the Near East from the 19th Century onwards., Dec 2004, Istanbul, Turquie. pp.140-155. halshs-00719862

\section{HAL Id: halshs-00719862 \\ https://shs.hal.science/halshs-00719862}

Submitted on 21 Jul 2012

HAL is a multi-disciplinary open access archive for the deposit and dissemination of scientific research documents, whether they are published or not. The documents may come from teaching and research institutions in France or abroad, or from public or private research centers.
L'archive ouverte pluridisciplinaire HAL, est destinée au dépôt et à la diffusion de documents scientifiques de niveau recherche, publiés ou non, émanant des établissements d'enseignement et de recherche français ou étrangers, des laboratoires publics ou privés. 


\section{Les quarantaines au Moyen-Orient : vecteurs ambigus de la modernité médicale $\left(\mathbf{X I X}^{\mathrm{e}}-\mathbf{X X}^{\mathrm{e}}\right.$ siècles $)$}

Les quarantaines ont été fondatrices d'une gestion collective de la maladie. Du point de vue de l'ordre politique, elles ont entraîné la mise en place d'un dispositif administratif impliquant une coordination par le pouvoir central. Du point de vue de l'ordre sanitaire, elles ont instauré une gestion policière de la maladie, et singulièrement de l'épidémie, ayant pour finalité l'enfermement et la surveillance des « suspects ». À partir de la seconde moitié du XIX ${ }^{\mathrm{e}}$ siècle, le dispositif quarantenaire, qui était demeuré jusqu'alors dans des cadres nationaux, s'inscrit dans un mouvement international qui entraîne le transfert, sur la rive sud de la Méditerranée, du système quarantenaire d'Europe, au moment où celui-ci, jugé obsolète, disparaît. Ce mouvement accompagne la montée en puissance des impérialismes et vient souligner ainsi les contours de la domination du Nord sur le Sud : le premier impose au second un dispositif très coercitif visant à arrêter la progression des épidémies vers l'Europe. Mais dans le même temps, le système quarantenaire établi au Moyen-Orient met à la disposition des usagers, des pèlerins musulmans pour la plupart, de nouvelles techniques médicales, bien souvent inaccessibles pour eux hors des limites du lazaret. On s'attachera ici à restituer les conditions de la mise en place du dispositif quarantenaire au Moyen-Orient, qui se joue dans le cadre de l'une des premières expériences d'internationalisation d'une politique publique, puis on décrira le système dans ses grandes lignes avant de chercher à cerner la façon dont était perçue par les pèlerins la double vocation de cette nouvelle génération de quarantaines, alliant contrainte et modernité médicale.

\section{D'une quarantaine à l'autre}

En Occident, le système séculaire de lutte contre les épidémies, dont le lazaret constitue la pièce maîtresse, commence à se lézarder au début du XIX ${ }^{\mathrm{e}}$ siècle sous les assauts de plus en plus pressants des intérêts économiques liés au commerce maritime, lequel pénètre alors dans une ère nouvelle grâce à la navigation à vapeur. Dès les années 1820-1840, la Grande-Bretagne, puis l'Autriche, adoptent des politiques de réduction des quarantaines sur leur territoire, suivis bientôt par la plupart des pays d'Europe. Certes les querelles scientifiques qui opposent contagionnistes et anticontagionnistes font rage tout au long du 
siècle ${ }^{1}$, jusqu'à ce que la bactériologie tranche finalement en faveur des premiers, mais quelle que soit la persistance des incertitudes, un mouvement inexorable se dessine en direction de la suppression des quarantaines.

Tous les acteurs de la scène internationale ne se rangent pourtant pas unanimement derrière ce choix. Bien des nuances se font jour, et pour certains, la pratique n'est pas toujours conforme à la théorie. Ainsi la Grande-Bretagne, qui défend ardemment des positions anticontagionnistes, entreprend précocement sur son sol de coûteux travaux d'assainissement qui rendent progressivement les quarantaines obsolètes ${ }^{2}$. En revanche, elle manifeste une plus grande sévérité quarantenaire dès lors qu'on s'éloigne des côtes des îles britanniques. Occupant l'Égypte en 1882, elle pénètre profondément les rouages du Conseil sanitaire, maritime et quarantenaire d'Alexandrie qui gère les lazarets de la zone du canal de Suez. C'est elle également qui prend en charge à partir de 1919, date de la disparition du Conseil sanitaire de Constantinople, le lazaret de l'île de Camaran, situé à l'entrée de la mer Rouge. En dépit de ses convictions et de la puissance des intérêts de la marine britannique, la GrandeBretagne participe activement, mais à ses propres conditions, au fonctionnement et à l'administration des quarantaines du Moyen-Orient.

Les Français, et plus généralement les Européens du sud, directement exposés à la menace épidémique venue de la rive sud de la Méditerranée, demeurent quant à eux plus attachés au principe de la quarantaine. Celui-ci s'assouplit néanmoins, et dès 1874 , lors de la Conférence sanitaire internationale de Vienne, les quarantaines terrestres sont jugées « inexécutables et inutiles ». Le mouvement se précipite ensuite et dans l'Europe issue de la révolution pastorienne, à la fin $\mathrm{du} \mathrm{XIX}^{\mathrm{e}}$ siècle, les quarantaines ont presque disparu ou sont réduites à un fonctionnement minime. À cette époque pourtant, la France se montre encore très active, notamment en la personne du professeur Adrien Proust ${ }^{3}$, pour organiser la défense quarantenaire de la zone du canal de Suez et de la mer Rouge. C'est encore l'obstination française qui conduit à la réouverture du dossier des quarantaines terrestres, pourtant clos depuis 1874, lors de l'inauguration de la ligne de chemin de fer du Hedjaz, en 1908.

\footnotetext{
${ }^{1}$ Sur cet aspect voir N. Howard-Jones, Les bases scientifiques des Conférences sanitaires internationales, 1851-1938, Genève, OMS, 1975.

${ }^{2}$ Le processus se fait cependant progressivement et les quarantaines ne disparaissent définitivement qu'en 1896. Voir Krista Maglen, "'The First Line of Defence' : British Quarantine and the Port Sanitary Authorities in the Nineteenth Century », Social History of Medicine, Vol. 15 (3), 2002, p. 413-428.

${ }^{3}$ Voir Daniel Panzac, Le docteur Adrien Proust. Père méconnu, précurseur oublié, Paris, L'Harmattan, 2003.
} 
De son côté l'Empire ottoman, qui considère pourtant avec beaucoup de réticences et de suspicion le développement d'un dispositif sanitaire international qui se déploie sur son territoire, dans la mesure où celui-ci menace directement sa souveraineté, défend ardemment les quarantaines qu'il considère comme la «prophylaxie du pauvre ${ }^{4}$. Faute de pouvoir entreprendre une véritable politique d'assainissement, les quarantaines apparaissent à ses yeux comme un moyen efficace, et relativement peu coûteux, d'assurer la sécurité sanitaire.

$\mathrm{Au}$ tournant des $\mathrm{XIX}^{\mathrm{e}}$ et $\mathrm{XX}^{\mathrm{e}}$ siècles, les quarantaines ne sont souvent plus qu'un souvenir en Europe même. Cependant, à cette même période, et avec le soutien de la plupart des acteurs internationaux, elles fleurissent sur la rive sud de la Méditerranée. Quarantaines d'Europe et d'Orient s'inscrivent en effet dans deux temporalités différentes. Implantées en Europe dès le XIV siècle, ce n'est qu'au début du XIX ${ }^{\mathrm{e}}$ siècle qu'elles font leur apparition en Orient. Muhammad Alî, vice-roi d'Égypte, fonde les premiers établissements quarantenaires sur la côte méditerranéenne du pays dans la décennie 1810. L’invasion du choléra, en 1831, l'incite ensuite à resserrer la surveillance. Avec son appui, les consuls généraux en poste à Alexandrie se constituent en Intendance de Santé publique, compétente pour toutes les questions quarantenaires. En 1839, une institution similaire, le Conseil sanitaire de Constantinople, est fondée dans la capitale ottomane. La création de ces deux conseils, qui auront en charge la gestion du système quarantenaire du Moyen-Orient, inscrit d'emblée celui-ci dans des structures à caractère international. En 1850, «la totalité de l'Empire ottoman est désormais ceinturée d'une ligne à peu près continue d'établissements sanitaires $\gg^{5}$ et à la fin du siècle, si les quarantaines apparaissent en Europe comme des institutions dépassées, elles sont bien vivantes en Orient où elle se présentent comme l'un des vecteurs de la modernité médicale.

La duplication sur la rive sud de la Méditerranée du système quarantenaire, entamée depuis les années 1810, connaît une brusque accélération en 1865. Cette année-là, le choléra change son itinéraire habituel de diffusion: abandonnant la voie terrestre qui longe la Caspienne et la mer Noire, il pénètre en Europe par la mer Rouge puis les ports méditerranéens de l'Égypte, accompagnant les pèlerins de retour de La Mecque. L'année suivante, une conférence internationale se réunit à Constantinople pour tenter d'assurer la

\footnotetext{
${ }^{4} \mathrm{~J}$-D Mizrahi, «Politique sanitaire et impérialisme à l'heure de la révolution pastorienne : le Conseil sanitaire de Constantinople (1892-1914)», Méditerranée, Moyen-Orient : deux siècles de relations internationales, W. Arbid, S. Kançal, J.D. Mizrahi et S. Saul (dir.), Paris, l'Harmattan, 2003.

${ }^{5}$ D. Panzac, La peste dans l'Empire ottoman 1700-1850, Louvain, Éditions Peeters, 1985, p. 491.
} 
sécurité sanitaire du pèlerinage musulman, devenue une préoccupation mondiale. Cette conférence, qui se sépare après sept mois de travaux sans aboutir à un accord sanctionné par une convention, pose néanmoins deux principes essentiels qui vont régir désormais un siècle de contrôle sanitaire international. Le premier est la mise en place, dans ses grandes lignes, du dispositif quarantenaire qui enserre la mer Rouge et la zone du canal du Suez, lequel donne naissance à une nouvelle génération de lazarets qui demeureront en usage jusqu'au cœur du $\mathrm{XX}^{\mathrm{e}}$ siècle. Le second est la désignation des pèlerins comme vecteurs principaux des maladies épidémiques, le choléra d'abord puis la peste qui fait sa réapparition au Hedjaz en 1897. Considérés comme particulièrement dangereux, ils seront soumis dès lors à un traitement d'exception, plus sévère que celui qui est réservé au commun des voyageurs.

Les participants à la conférence de 1866 s'accordent sur le fait que les mesures quarantenaires, jugées efficaces, le sont d'autant plus qu'elles sont appliquées à proximité du foyer primitif de la maladie. Le choléra venant d'Inde, on doit donc d'abord chercher à en bloquer la diffusion en instituant à l'entrée de la mer Rouge, en un lieu adéquat, si possible une île, un établissement sanitaire où tous les navires seraient assujettis à une quarantaine. Par ailleurs, un service sanitaire doit être organisé dans les ports et échelles du littoral de la mer Rouge, et notamment au Hedjaz même, de façon à renseigner la communauté internationale sur l'état sanitaire de la région, à délivrer ou viser les patentes de santé et à veiller à l'exécution des mesures hygiéniques qui seraient prescrites. Enfin, dans l'éventualité où le choléra pénètrerait malgré tout au Hedjaz, il convient d'empêcher son extension vers l'Égypte, et au-delà en Europe, en établissant dans le golfe de Suez une seconde ligne de défense constituée principalement de deux lazarets, l'un destiné aux provenances ordinaires et le second aux navires de pèlerins.

Le scénario adopté lors de la conférence de 1866 se maintiendra dans ses grandes lignes pendant près d'un siècle. Il sera certes amélioré, modifié à la marge et surtout de plus en plus juridiquement encadré, mais il ne subira pas de changements fondamentaux. Cette continuité témoigne de la volonté politique qui se manifeste d'emblée sur la question du contrôle sanitaire du pèlerinage, dans la mesure où un début de réalisation concrète de ce dispositif est entrepris alors même que les débats scientifiques qui sous-tendent ces choix ne sont pas encore tranchés, et qu'aucune contrainte législative n'existe, avant 1892, pour la motiver. Les principes adoptés à Constantinople en 1866 vont cependant peu à peu influencer certaines législations nationales, qui prescrivent dès lors des recommandations visant à assurer une meilleure sécurité sanitaire au pèlerinage et de meilleures conditions de voyage 
aux pèlerins. Les stations quarantenaires d'al-Wajh et de Tor ${ }^{6}$, dans le golfe de Suez, sont mises en œuvre dès les années 1870, puis le lazaret de Camaran, verrou d'entrée de la mer Rouge, fonctionne pour la première fois lors du pèlerinage de 1882.

Une seconde ligne de force adoptée en 1866, et qui ne se démentira pas, est l'attention privilégiée portée aux pèlerins dont l'encadrement sanitaire sera de plus en plus précisé et codifié par la législation internationale adoptée à partir de la Conférence sanitaire internationale de Venise en 1892. Celle-ci distingue en effet les navires ordinaires des navires de pèlerins (on disait alors les «navires à pèlerins »), lesquels sont soumis à une aggravation des mesures d'ordre général. Il est vrai que les pèlerins ont la malchance de venir nombreux des zones contaminées et qu'ils voyagent en masse dans des conditions souvent déplorables. Les voyageurs ordinaires, quant à eux, sont bien moins suspects d'être porteurs de maladies épidémiques dans la mesure où ils vivent et voyagent dans des conditions infiniment plus décentes. Ainsi le lazaret de Camaran, d'abord prévu pour tous les navires pénétrant dans la mer Rouge, n'y recevra finalement que les pèlerins. D'ailleurs, si tous les pèlerins sont soigneusement filtrés à l'entrée et à la sortie de la mer Rouge, une plus grande tolérance se manifeste à l'égard des navires ordinaires ou de transport de troupes. Ainsi, en 1886, le navire de troupe français le Canton, retour d'Extrême-Orient porteur du choléra, est autorisé à limiter son temps d'observation à Tor puis à franchir le canal de Suez en quarantaine, c'est-à-dire sans aucun contact avec l'extérieur. La France s'en félicite d'autant plus que, comme le souligne un diplomate, «les passagers n'auraient pas pu séjourner sans péril, au milieu des groupes de pèlerins qui encombrent actuellement le campement de Tor $»^{7}$. C'est d'ailleurs pour éviter cette promiscuité jugée fâcheuse qu'on réitère, dans les années 1885-86, l'idée émise à Constantinople de fonder un lazaret destiné aux provenances ordinaires. Mais la motivation demeure faible dans la mesure où le danger épidémique lié aux voyageurs ordinaires apparaît peu important. Aussi, lorsqu'en 1896 la peste fait sa réapparition en Inde, et que toutes les provenances de ce pays sont soumises à quarantaines, les passagers sont-ils conduits à la station sanitaire des Sources de Moïse. Mais rien n’est prévu pour les accueillir, il ne s'y trouve même pas un lazaret. En revanche, l'inquiétude permanente que suscitent les pèlerins motive la communauté internationale à se mobiliser encore, dans le premier tiers du

\footnotetext{
${ }^{6}$ Initialement, la station d'al-Wajh est destinée aux pèlerins et celle de Tor aux provenances ordinaires. Après 1881, l'arrangement inverse est adopté mais la station d'al-Wajh demeure quasi virtuelle.

${ }^{7}$ Lettre du consul de France à Alexandrie adressée à M. Freycinet, président du Conseil et ministre des Affaires étrangères, en date du 27 septembre 1886. Centre des Archives Diplomatiques de Nantes, fonds Unions Internationales, ${ }^{\text {er }}$ versement, carton 505.
} 
$\mathrm{XX}^{\mathrm{e}}$ siècle, pour créer promptement de nouveaux lazarets et stations sanitaires sur les itinéraires terrestres lorsque les pèlerins se mettent à emprunter le chemin de fer puis la route pour se rendre à La Mecque. Les pèlerins subissent donc un traitement particulier qui dépasse en sévérité celui appliqué à une autre masse de voyageurs jugée dangereuse, les immigrants. Ainsi le service quarantenaire d'Ellis Island, dans la baie de New York, de triste réputation avant la Première Guerre mondiale, ne retient plus que quelques milliers d'étrangers dans les années $1930^{8}$, alors que les quarantaines de la mer Rouge sont encore en pleine activité. À l'heure où la plupart des voyageurs n'y sont plus soumis, le système quarantenaire pèse encore lourdement sur les pèlerins se rendant à La Mecque ou en revenant et impose au voyage sacré une halte obligée où les pèlerins subissent une surveillance sanitaire impliquant une certaine violence matérielle et symbolique.

\section{La pratique quarantenaire : contrainte et innovation}

Les décisions abstraites prises par des aréopages de médecins et de diplomates réunis en conférences internationales, la plupart du temps dans les capitales européennes, ont en effet un impact direct et très concret sur le déroulement du pèlerinage musulman. Celui-ci a toujours constitué un voyage long et dangereux. Longtemps les caravanes ont joué le rôle de quarantaine naturelle en raison de la durée du voyage. L'accélération de ce dernier, grâce aux moyens de transport modernes, impose de substituer à ce processus naturel des établissements ad hoc à caractère contraignant, voire coercitif, que les voyageurs occidentaux et les acteurs liés aux intérêts du commerce international fustigeaient pourtant depuis longtemps dans leur version européenne.

La mise en place du dispositif quarantenaire sur la rive sud de la Méditerranée fut longue et souvent laborieuse, dans la mesure où elle impliquait un grand nombre d'acteurs internationaux, peu rompus encore aux règles de la négociation internationale dont le cycle des conférences sanitaires constitue précisément l'un des épisodes fondateurs. Tout au long de la seconde moitié du $\mathrm{XIX}^{\mathrm{e}}$ siècle, le système quarantenaire ne cesse pourtant de se perfectionner. Jusque dans les années 1890, il relève largement du bricolage, progresse à coup d'initiatives peu coordonnées et souffre d'un manque de moyens financiers et de personnels. D'ailleurs, le système et ses imperfections soulèvent bien des critiques. Pour être efficace, ce

\footnotetext{
${ }^{8}$ R. Dujarric de la Rivière, Prophylaxie nationale et internationale des maladies épidémiques, Paris, Éditions médicales Flammarion, 1948, p. 203. Notamment en raison de la sélection effectuée avant l'embarquement, il n'y a plus que 4288 migrants retenus quelques jours à Ellis Island en 1933. À cette même date, les lazarets de Tor et de Camaran reçoivent chacun 20 à 30000 pèlerins durant la campagne quarantenaire.
} 
système se devrait en effet d'être parfaitement étanche. Or les conditions approximatives de sa mise en œuvre rendent cette étanchéité improbable et les divers pays impliqués, ainsi que les structures internationales de santé, notamment les Conseils d'Alexandrie et de Constantinople, ne cessent de se renvoyer la responsabilité des manquements, lançant des accusations croisées d'incompétence et de négligence.

Cependant l'adoption de la première convention sanitaire internationale, en 1892, offre un cadre juridique désormais stable pour poursuivre l'implantation du dispositif quarantenaire en Méditerranée orientale, et lors de la conférence de Venise, en 1897, la rénovation et la modernisation des lazarets de la mer Rouge sont expressément demandées. D'importants travaux sont entrepris dans les stations de Camaran et de Tor et au tournant du siècle, ces établissements présentent le paradoxe d'être des structures régies par des principes d'isolement et de contrainte jugés dépassés partout ailleurs, mais qui accueillent dans le même temps des technologies innovantes qui en font des vecteurs ambigus de la modernité médicale. La description d'une séquence quarantenaire idéale ${ }^{9}$ au lazaret de Tor, considéré comme le plus moderne qui soit alors, permet de saisir cette ambivalence.

Chaque année, au moment du pèlerinage, deux cas de figure peuvent se présenter : soit le pèlerinage est net, c'est-à-dire qu'aucune maladie épidémique ne s'y est manifestée, soit il est brut quand des cas de choléra ou de peste ont été déclarés. Dans le premier cas, la halte au lazaret de Tor des pèlerins de retour, transitant par l'Égypte, est de courte durée, trois jours environ, avec désinfection des hommes et des bagages et examen médical. Dans le second cas, la quarantaine peut s'éterniser plusieurs semaines. Ainsi en 1893, année d'une terrible épidémie de choléra au Hedjaz, qui aurait causé la mort d'un tiers des pèlerins présents sur le territoire sacré, la durée du séjour dans les sections de Tor a varié de 15 à 50 jours, auxquels il convient d'ajouter la deuxième quarantaine dans un lazaret secondaire ${ }^{10}$. Les années brutes, le gain de temps de voyage obtenu grâce aux transports modernes se trouve annulé. Ce n'est

${ }^{9}$ Cette description s'appuie essentiellement sur les sources suivantes: Dr Noury Pacha, Retour des pèlerins en 1893. Service quarantenaire de Tor. Rapport au Conseil supérieur de santé, Constantinople, 1893; R. Dujarric de la Rivière, Prophylaxie nationale et internationale des maladies épidémiques, Paris, Éditions médicales Flammarion, 1948 ; G. Zananiri, Tor historique et quarantenaire, Alexandrie, CSMQ, 1925 ; Dr. Delarue, Le pèlerin de La Mecque. Son hygiène, ses maladies, Paris, Librairie J-B Baillière et Fils, 1892 ; M. Boniteau «Le pèlerinage à La Mecque et les campements quarantenaires », Bulletin de la Société de Géographie d'Égypte, Tome 5, 1902, p. 647-667 ; T. Barthas, «Le retour de La Mecque. El Tor», La Revue de Paris, n5, 1914, p. 85-118. En réalité, les choses ne se passent bien entendu jamais comme les promoteurs du projet l'ont prévu (voir infra.)

${ }^{10}$ Les puissances coloniales astreignent en effet les pèlerins relevant de leur autorité à une ultime quarantaine avant de débarquer dans leur pays d'origine. 
qu'à partir des années 1920 et surtout 1930, avec le recul du péril épidémique, que les temps d'observation et de quarantaine seront sensiblement diminués. Mais de 1891 à 1918, date à laquelle le choléra disparaît du Hedjaz, on ne compte que six années nettes sur vingt-huit ; la plupart des pèlerins qui font le voyage sacré au cours de cette période sont donc amenés à connaître le lazaret.

Les bateaux chargés de pèlerins arrivant en rade de Tor se voient attribués un mouillage. Ils sont aussitôt visités par le médecin de la quarantaine qui prend connaissance de la patente et du nombre des pèlerins à bord, puis repère les malades, lesquels sont conduits à l'hôpital, avant de donner l'autorisation de débarquer. La plupart du temps le débarquement s'effectue à l'aide de barques, mais à Camaran les pèlerins doivent parfois se jeter à la mer pour rejoindre le rivage. La plupart des pèlerins rejoignent ensuite à pied les bâtiments de désinfection. Seuls les femmes, les vieillards et les personnes affaiblies sont pris à bord du chemin de fer sur voies Decauville qui transporte les bagages.

À l'entrée des bâtiments de désinfection, les pèlerins remettent leurs passeports à l'administration, qui les conserve jusqu'au moment du départ, et s'acquittent de la taxe sanitaire. On procède ensuite à la vérification et au tri des bagages. Il s'agit d'une opération longue et fastidieuse car les pèlerins voyagent très lourdement chargés; le voyage sacré s'accompagne en effet souvent d'activités commerciales. Un jeton marqué d'un numéro correspondant à chaque bagage déposé au service du triage est remis aux pèlerins.

Dans les années 1890, outre les bagages, seuls les vêtements portés par les pèlerins étaient soumis à la désinfection et les pèlerins eux-mêmes invités à procéder à un simple bain de mer. Au tournant du siècle, des douches à eau de mer sont introduites dans les lazarets, ainsi que des bains pour les voyageurs de première classe. Les pèlerins sont tenus de se déshabiller entièrement avant de prendre une douche savonnée, laquelle est parfois accompagnée d'un épouillage. Les vêtements portés sont placés dans des sacs pourvus de numéros correspondant à ceux des jetons remis aux pèlerins, puis vêtements et bagages sont désinfectés au moyen de grandes étuves à vapeur produisant une chaleur de 110 degrés apte à détruire les germes. L'administration du lazaret fournit ensuite à chacun une longue chemise (galabeyya) portée jusqu'à ce que les pèlerins, munis de leur numéro, récupèrent leurs effets et leurs biens.

Les pèlerins sont ensuite conduits vers les sections (ou divisions), lesquelles sont généralement organisées par bateau et étroitement surveillées par des soldats pour empêcher toute communication avec l'extérieur ou entre les sections. À Tor, les pèlerins sont hébergés sous des tentes. On en compte 1300 en 1890, formant dans le désert du Sinaï une éphémère 
ville de toile. À partir du début du $\mathrm{XX}^{\mathrm{e}}$ siècle, elles sont peu à peu remplacées par des bâtisses en dur. Chaque section est traversée d'une enceinte de fil de fer qui sépare les pèlerins de première et de seconde classe. L'approvisionnement du campement est assuré par des entrepreneurs locaux qui ont obtenu des concessions. Les prix sont en principe contrôlés, en réalité prohibitifs. L'ensemble des bâtiments et des sections est relié par une voie de chemin de fer Decauville. En 1902, l'électricité est installée sur l'ensemble du campement et dès 1914, toutes les communications internes se font par téléphone. Il existe en outre un télégraphe pour les communications externes. L'eau est prélevée dans des puits et des canalisations distribuent le précieux liquide dans les sections au moyen de bornes-fontaines munies de robinets. Les sections comprennent des magasins, « un genre de café où peuvent se réunir les pèlerins ${ }^{11}{ }^{1}$, des fours. Pour les pèlerins, le temps de la quarantaine s'écoule en discussions et pieuses lectures.

La vie du campement est néanmoins scandée par les nécessités de l'observation médicale à laquelle sont soumis les pèlerins. En principe, les médecins du lazaret assurent une visite médicale quotidienne auprès de chaque pèlerin hébergé dans les sections. En outre, à partir du début du $\mathrm{XX}^{\mathrm{e}}$ siècle, les examens bactériologiques se multiplient et la vaccination anticholérique et antivariolique est introduite. Il existe enfin quatre hôpitaux à Tor, pour les maladies infectieuses, les femmes, la chirurgie et la dysenterie. Les personnes atteintes de choléra ou de peste sont isolées dans une section spéciale puis, à partir de 1912, dans des pavillons d'isolement. Au terme de leur contumace, les pèlerins rejoignent leur bateau, lequel a subi également une désinfection totale, laissant derrière eux de nombreux morts, plus souvent victimes de maladies ordinaires ou d'épuisement que des épidémies dont la crainte a suscité l'implantation d'un tel système ${ }^{12}$.

Sans qu'on puisse les réduire à cela, ne serait-ce qu'en raison du fait que la réclusion y est très temporaire, les quarantaines présentent certains traits des institutions totales. Trains, mise à nu, douches, ne peuvent manquer d'évoquer les camps de concentration. Le système quarantenaire est un dispositif coercitif qui agit de façon très forte et contraignante sur les corps des pèlerins qu'il trie, étrille, déshabille et enferme. Pourtant, dans ce contexte particulier emprunt d'une certaine violence, les pèlerins sont également placés en présence

\footnotetext{
${ }^{11}$ Zananiri, op. cit., p. 89.

${ }^{12}$ Durant la campagne quarantenaire de 1890, d'une durée de trois mois, 422 décès sont enregistrés au campement de Tor, dont 135 par choléra et 287 par maladie ordinaire : Dr Catelan, Rapport sur le choléra au Hedjaz en 1890, Melun, Imprimerie administrative, 1892, p. 4 .
} 
des outils de la modernité médicale, qu'ils accueillent avec résignation sinon bienveillance, mais parfois aussi de façon critique.

\section{La quarantaine, lieu de rencontre avec la modernité médicale}

Si les textes rendant compte des mécanismes internationaux par lesquels ont été mises en place les quarantaines du Moyen-Orient sont nombreux, il n'en va pas de même des témoignages émanant des usagers de ces structures, médecins et surtout pèlerins. À travers les rapports des médecins quarantenaires et les quelques récits de pèlerinage (rihlât) écrits au tournant des $\mathrm{XIX}^{\mathrm{e}}$ et $\mathrm{XX}^{\mathrm{e}}$ siècles, on peut néanmoins saisir une image de la façon dont les quarantaines étaient perçues et vécues par ceux qui y étaient astreints et de la manière dont se faisait la rencontre entre les pèlerins et les pratiques médicales et sanitaires auxquelles ils étaient soumis.

Les quarantaines ont une vocation sécuritaire. Nées de la crainte d'une expansion incontrôlée des épidémies qui pourraient menacer l'Europe, elles sont aussi instrumentalisées par les puissances coloniales pour exercer un contrôle, un filtrage de leurs sujets musulmans qu'elles soupçonnent d'aller puiser à La Mecque l'inspiration politique visant à une remise en cause de leur pouvoir. En revanche, alors que la période est profondément influencée par les principes de l'hygiénisme, il ne semble pas y avoir eu de volonté pédagogique, du moins explicite, dans le dispositif quarantenaire. Il s'agissait avant tout de purifier le pèlerin, de le rendre indemne de toute maladie avant de le laisser poursuivre sa route. L'introduction de technologies médicales innovantes (vaccination, examens bactériologiques) vise une meilleure efficacité et non l'apprentissage. Malgré tout, les lazarets constituent le lieu privilégié d'une rencontre directe entre les pèlerins et la médecine moderne, à l'heure où dans leurs pays respectifs l'implantation de cette dernière est encore très restreinte.

La période pendant laquelle les lazarets de Tor et de Camaran sont mis en activité pour recevoir les pèlerins est désignée sous le terme de «campagne quarantenaire », ce qui souligne sa vocation de lutte, de quasi-guerre engagée contre les germes infectieux. Ces lazarets ne sont en effet pas permanents, contrairement à ceux qui sont destinés à recevoir les provenances ordinaires. Chaque année, ces deux territoires désertiques s'animent pour une durée de trois à six mois, se transformant en villes éphémères et autosuffisantes. En 1925, Tor reçoit pour les besoins de son fonctionnement une équipe de 137 permanents, auxquels s'ajoute une moyenne de 371 journaliers (gardes, préposés à l'approvisionnement...) ${ }^{13}$. C'est

\footnotetext{
${ }^{13}$ Zananiri, op. cit., p. 97
} 
dès lors, selon Thérèse Barthas, «la rencontre imprévue de notre génération scientifique qui vient de naître et du vieux monde que des milliers d'années n'ont guère changé ; c'est le contraste de nos blouses stérilisées, de nos étuves perfectionnées, de nos microscopes, avec les haillons, les paquetages de tapis centenaires et d'étoffes rares de nos clients malgré eux cheikhs vénérables que l'on douche, harems affolés qu'on expose au soleil, Bédouins que l'on emploie à vaporiser des antiseptiques... $»^{14}$.

L'ordonnancement de ces villes éphémères suit un plan qui respecte les principes de l'hygiène. Accueillant les «suspects » dès leur arrivée, les bâtiments de désinfection se situent près du rivage. Au-delà, à l'intérieur des terres, s'étendent les sections et les structures hospitalières. Toute la partie qui se situe en amont du service de la désinfection est qualifiée de partie sale ou contaminée ; lorsque les pèlerins ont subi la douche et la désinfection, en quittant le bâtiment par l'arrière, ils passent une frontière immatérielle qui les mène à la partie propre du lazaret. Les services de désinfection fonctionnent ainsi comme une gigantesque machine qui avale les pèlerins puis les recrache lavés, purifiés, inoffensifs. Au cœur de la séquence religieuse que forme le pèlerinage à La Mecque, la halte au lazaret impose une formule de la pureté qui se pose en alternative à la pureté d'ordre religieux. Les ablutions rituelles jouent un rôle majeur au cours du pèlerinage, en raison de la nécessité de la purification. Le système de la quarantaine propose quant à lui une purification radicale et sa rationalité scientifique ${ }^{15}$ ne tient aucun compte des formes religieuses de la pureté. Tentant avec énergie de mettre en place un ordre régi par la salubrité et la sécurité sanitaire, il redessine sans états d'âme les frontières entre le pur et l'impur. Les besoins religieux des pèlerins sont d'ailleurs totalement ignorés, et ce n'est que très tardivement que l'on songe à construire des mosquées dans l'enceinte du lazaret. En 1925, elles ne sont que deux, construites tout récemment ${ }^{16}$.

Ces principes de nature hygiéniste ne s'implantent cependant pas sur un terrain vierge. En ce tournant de siècle, le discours hygiéniste a déjà pénétré dans les pays du sud, souvent dans les bagages du colonialisme, discours relayé localement, notamment en Égypte, par le

\footnotetext{
${ }^{14}$ T. Barthas, art. cit., p. 86.

${ }^{15}$ Celle-ci apparaît de façon évidente dans les rapports des médecins et responsables de quarantaine qui privilégient les développements statistiques et les considérations épidémiologiques.

${ }^{16}$ Zananiri, op . cit., p. 91. Il y a alors vingt sections dans le lazaret, et toute communication est interdite entre les sections.
} 
réformisme ${ }^{17}$. Les intellectuels modernistes égyptiens qui effectuent le pèlerinage à cette époque ont intégré, assimilé et repris à leur compte ce discours hygiéniste qu'ils entendent diffuser auprès de leurs concitoyens afin d'effacer le « retard de civilisation » dont leur pays est victime. Ils perçoivent donc d'un œil averti et critique les pratiques hygiénistes du lazaret, et les jugent finalement assez peu opératoires. La plupart des récits convergent vers une mise en cause de la façon dont sont réalisées les procédures guidées par le souci hygiéniste et celui de la sécurité sanitaire. En revanche, on ne relève nulle part de mise en cause du principe même de la désinfection, de la douche ou des traitements médicaux.

La belle ordonnance du lazaret décrite plus haut n'a en effet pas de réalité. Face à la difficulté à gérer la foule, les opérations se déroulent souvent dans le désordre, voire le chaos. L'entassement dans les tentes, l'inconfort, la pénurie des denrées sont maintes fois relevés et la pagaille atteint souvent son comble lors des manœuvres de débarquement et d'embarquement. Le mélange des sexes au cours des opérations de désinfection est jugé particulièrement scandaleux, a fortiori dans le cadre du voyage sacré. Certes il est prévu qu'hommes et femmes se déshabillent dans deux pièces séparées mais à Tor, en 1893, la salle de déshabillage des femmes ne pouvant accueillir que 4 à 5 personnes au plus, les pèlerins des deux sexes se côtoient dans la pièce principale ${ }^{18}$. L'administration quarantenaire fournit ensuite aux pèlerins un vêtement leur permettant d'attendre la récupération de leurs effets dans la cour, où ils sont longuement exposés à la chaleur ou aux intempéries, tête et pieds nus. Rifa'at Pacha, un officier égyptien qui accompli le pèlerinage à plusieurs reprises, assiste scandalisé à une telle scène en 1903 et demande à ce que les pèlerins soient mieux vêtus et protégés au sortir de la douche, précisément pour respecter les conditions élémentaires d'hygiène. Le même Rifa'at Pacha décrit avec dégoût l'état des latrines de Tor, aussi éloignées que possible des notions d'hygiène et qui ne peuvent selon lui que jouer un rôle néfaste de diffusion des maladies ${ }^{19}$. Promoteurs des quarantaines et usagers se renvoient donc le discours sur l'hygiène, les premiers l'imposant aux seconds dans le but de les débarrasser de tout péril épidémique, et les seconds soulignant les insuffisances du système mis en place par les premiers qui n'adopteraient pas les «comportements civilisés » qu'ils prônent par ailleurs.

\footnotetext{
${ }^{17}$ Sur cette question voir A. Roussillon (dir.), Entre Réforme sociale et mouvement national. Identité et modernisation en Égypte (1882-1962), CEDEJ, Le Caire, 1995.

${ }^{18} \mathrm{H}$. Bitter, Rapport sur une inspection des campements quarantenaires de El-Tor et de Ras Mallap, Alexandrie, Imprimerie générale, 1893, p. 28-29.

${ }^{19}$ Rifa'at P acha, Mi'rât al-Haramayn, Beyrouth, Dar al-Ma'arifa, s.d., p. 31-32.
} 
Les lazarets sont particulièrement exemplaires de la tension entre vocations collective et vocation individuelle de la médecine et des difficultés à gérer chacune d'elle, et plus encore les deux ensemble. Bien des opérations effectuées au sein du lazaret sont de nature collective (douche, désinfection...) mais d'autres, de plus en plus nombreuses quand on s'avance dans le $\mathrm{XX}^{\mathrm{e}}$ siècle, visent les individus (visite médicale, vaccination, examen bactériologique...). Or au lazaret, se manifestent pleinement les difficultés à trouver des modalités efficaces pour cette médecine individuelle dans un contexte de masse. Ce sont en effet plusieurs milliers de pèlerins qui sont réunis au même moment au lazaret, pour une équipe médicale somme toute réduite. Dès lors, et bien des observateurs le déplorent, la visite médicale se réduit souvent à un simple coup d'œil jeté à un visage, accompagné d'une furtive prise de pouls. Outre la routine des examens journaliers, le lazaret offre à certains une rencontre plus étroite avec la médecine : les accouchements y sont médicalisés et certains pèlerins, identifiés comme malades, sont admis à l'hôpital où ils subissent un traitement particulier, voire une opération. Aussi, malgré les imperfections du système, le lazaret met-il l'usager en rapport quasi quotidien avec le médecin. Certes la rencontre se fait mal, le dialogue s'engage difficilement, bien souvent simplement faute d'une langue commune. D'ailleurs, de nombreux pèlerins déplorent que les médecins de quarantaine soient pour la plupart des étrangers ignorant l'arabe, langue parlée par une grande partie des usagers de la quarantaine.

En dépit des difficultés de communication, les pèlerins ne manifestent pas d'hostilité directe à l'égard des actes médicaux eux-mêmes. Ils les subissent le plus souvent avec résignation, bien qu'ils n'en comprennent guère le sens. Le docteur Thérèse Barthas, qui participe à la campagne quarantenaire de Tor en 1914, fait ainsi le récit d'une opération sur des ulcères à la jambe subie à l'hôpital du lazaret par « un noble seigneur du Turkestan ». Pas de communication verbale possible, bien sûr, aussi « deux robustes gaillards » l'empoignentils pour l'étendre «sur un chevalet de forme bizarre » alors que trois dames portant d'étranges instruments s'approchent. Rien pourtant de « cette pantomime effarante » ne semble émouvoir ou révolter ce pèlerin venu d'une contrée si lointaine. Mais endormi au masque avec du chloroforme, promptement opéré, l'homme en se réveille pas. Inquiète, l'équipe médicale tente de le sortir du sommeil par des appels, des cris, des «frictions énergiques, compresses d'eau froide, secousses en avant et en arrière ». Rien n'y fait jusqu'à ce qu'une doctoresse l'interpelle d'un puissant « Hadji baba !», son nouveau titre si prestigieux, qui le tire enfin de 
sa torpeur. Réveillé depuis longtemps, les traitements qu'on lui infligeait ne l'avaient pas plus ému que toute l'agitation étrange qui avait précédé l'opération ${ }^{20}$.

Cette résignation à l'égard des actes médicaux est beaucoup moins présente vis-à-vis des problèmes liés au quotidien de la vie au lazaret. En ce qui concerne les aspects médicaux, les récriminations ne portent pas sur le principe des actions, mais sur les modalités de leur réalisation. Le mépris, le manque de considération à l'égard des pèlerins, la négligence avec laquelle on traite leurs affaires (les bagages, notamment, sont souvent considérablement endommagés par l'opération de désinfection) soulèvent maintes protestations. Et celles-ci sont plus vives encore quand il s'agit des aspects directement matériels de la vie au lazaret. Le prélèvement des taxes sanitaires est souvent un sujet de vives tensions. Dans certains cas, quand le lazaret manque de pain ou que les provisions sont trop coûteuses, on frôle l'émeute ${ }^{21}$. Ce n'est jamais la modernité médicale, ni même la modernité de façon générale, qui est refusée par les pèlerins. Ainsi, bien que la plupart d'entre eux ne sachent ni lire ni écrire, ils utilisent très largement le télégraphe de Tor pour prévenir leur famille de leur arrivée prochaine et les rassurer. En revanche, le principe même de l'enfermement, qui prive les gens de liberté, les détourne de leurs activités et entraîne des procédures peu attentives aux droits humains, est unanimement rejeté. En témoigne la persistance, jusque dans les années 1950, des filières de clandestinité qui permettent d'éviter le lazaret par des traversées de la mer Rouge en barque ou grâce à d'autres stratégies, dès lors qu'on a l'argent pour payer.

Les quarantaines du Moyen-Orient, fondées dans un mouvement d'ampleur internationale à la fin du $\mathrm{XIX}^{\mathrm{e}}$ et au début du $\mathrm{XX}^{\mathrm{e}}$ siècles, reposent toujours sur le principe fondateur de l'expérience au Moyen Âge, celui de l'enfermement. Mais en cette période dominée par la bactériologie et les progrès médicaux, les quarantaines se chargent de nouveaux sens. Les usagers ne se contentent plus d'y attendre leur libération dans un morne ennui, ils sont soumis désormais, durant leur séjour, à des procédures d'ordre médical et sanitaire. Mais comme les précédentes, cette nouvelle génération de lazarets provoque le rejet des usagers, qui tentent dans la mesure du possible d'y échapper. Pour les pèlerins musulmans, qui en sont les principaux, sinon les seuls destinataires, les quarantaines interrompent inopinément le retour du voyage sacré. En outre, ils sont confrontés, souvent assez brutalement, aux dysfonctionnements d'un système qui ne peut tenir ses promesses de

\footnotetext{
${ }^{20}$ T. Barthas, art. cit., p. 114-115.

${ }^{21}$ Ce fut le cas à Ras Mallap en 1893. Voir H. Bitter, op.cit. p. 43.
} 
contrôle face à l'énormité des foules mouvantes à traiter. Pour autant, ce ne sont pas les procédures sanitaires qui sont dénoncées, mais le principe d'enfermement lui-même, la privation de liberté et le manque de considération pour l'individu et ses biens. Bien que les puissances coloniales soient largement à l'origine de la mise en place du dispositif quarantenaire au sud de la Méditerranée, celui-ci ne peut cependant être considéré comme une institution purement coloniale, ne serait-ce qu'en raison du fait que l'Empire ottoman y participe également jusqu'à sa disparition. D'ailleurs, les pèlerins commencent confusément à percevoir qu'un tel dispositif relève de la responsabilité d'un ordre international abstrait, encore en gestation, qui vient à son tour, à la suite des États, chercher à contrôler les mouvements et la vie des hommes. 